\title{
A Comparison of Magnetite Particles Produced Anaerobically by Magnetotactic and Dissimilatory Iron- reducing Bacteria
}

Bruce M. Moskowitz

Department of Geology, University of California-Davis, Davis, Ca 95616

Richard B. Frankel

Physics Department, California Polytechnic State University, San Luis Obispo, Ca 93407

Dennis A. Bazylinski and Holger W. Jannasch

Department of Biology, Woods Hole Oceanographic Institution, Woods Hole, MA 02543

Derek R. Lovley

US Geological Survey, Reston, VA 22092

\section{ABSTRACT}

We compare the magnetic properties of fine-grained magnetite produced by two newly isolated anaerobic bacteria, a magnetotactic bacterium (MV-1) and a dissimilatory iron-reducing bacterium (GS15). Although room-temperature magnetic properties are generally different between the two microorganisms, MV-1 and GS-15 magnetites can be most easily distinguished by the temperature variation of saturation remanence obtained at liquid helium temperatures. Magnetite produced by MV1 displays a sharp discontinuity in intensity at $100 \mathrm{~K}$ related to the Verwey transition. Magnetite produced by GS-15 displays a gradual decrease in intensity with temperature due to the progressive unblocking of magnetization. The differing behavior is due exclusively to different grain size distributions produced by these microorganisms. MV-1 produces magnetite with a narrow grain size distribution that is within the stable single domain size range at room temperature and below. GS-15 produces magnetite 
with a wide grain size distribution extending into the superparamagnetic (SPM) size range. Our results show that a substantial fraction of particles produced by GS-15 are SPM at room temperature.

\section{INTRODUCTION}

Magnetite produced by bacteria and other microorganisms may make a significant contribution to the natural remanent magnetization of sediments [e.g., Vali et al., 1987]. Deposition of intracellular magnetite by magnetotactic bacteria in membrane-bound vesicles called magnetosomes [Frankel et al., 1979; Bazylinski et al., 1988] is now the only known process by which microorganisms produce magnetite in natural sediments. Deposition of extracellular magnetite particles produced by dissimilatory iron-reducing bacteria could also be another source of magnetite in natural sediments [Lovley et al., 1987]; however, magnetite produced by these microorganisms has been isolated only under laboratory conditions. Various species of magnetotactic bacteria inhabit aerobic and anaerobic sediments and produce permanent, single-magnetic domain (SO) particles with narrow size distribution and species specific morphology. The magnetosomes are often arranged in intracellular chains [Blakemore, 1982]. When cells die and lyse, chains of magnetite particles with their enveloping membrane can be deposited as intact units in the sediment, or the chains can disassemble and the particles be deposited individually. Isolated chains of SD sized magnetite particles with similar biogenic morphologies have been found in several Quaternary and Tertiary deep-sea sediments [Petersen et al. , 1986; Vali et al., 1987]. Dissimilatory ironreducing bacteria inhabit anaerobic sediments and under laboratory conditions produce extracellular fine grained magnetite as a byproduct of ferric iron respiration. The particles are not enveloped, do not have unique morphology, and are not arranged in any particular configuration; however, magnetite production can be copious [Lovley et al., 1988].

Until recently, magnetotactic bacteria were believed to occur only in microaerobic aquatic environments because of an apparent requirement for molecular oxygen for magnetite production 
[Blakemore et al., 1985]. Aquaspirillum magnetotacticum (MS-1), available in pure culture, is a typical example [Blakemore et al., 1979]. The magnetic properties of magnetite produced by MS-1 have been described by Moskowitz et al. [1988]. Two other species of magnetite producing bacteria have now been isolated and cultured under anaerobic conditions. The first, a magnetotactic bacterium, designated MV-1, was isolated from sulfide-rich sediments of an estuarine salt marsh [Bazylinski et al., 1988]. Individual magnetite particles in MV-1 are parallelipipeds with approximate dimensions 40x40x60 nm [Bazylinski et al., 1988]. The other bacterium is not magnetotactic, but a dissimilatory iron-reducing bacterium, designated GS-15 [Lovley et al., 1987]. Production of magnetite by anaerobic bacteria could be an importance source of magnetite in suboxic marine sediments within the zone of iron reduction [Karlin et al.,1987]. In this paper we compare the magnetite properties of magnetite particles produced by MV-I with those from GS-15. We show that whereas ambient temperature magnetic properties are insufficient to distinguish clearly between magnetite produced by these two microorganisms, they can be distinguished by their low temperature magnetic properties.

\section{EXPERIMENTAL PROCEDURE}

Strain MV-1 was grown in batch culture medium under anaerobic conditions in a diluted artificial sea water medium with nitrous oxide as the terminal electron acceptor (D.A. Bazylinski and H.W. Jannasch, unpublished data). Cells were harvested by centrifugation at $4^{\circ} \mathrm{C}$ and washed several times with $50 \mathrm{mM}$ potassium phosphate buffer containing $18 \mathrm{~g} \mathrm{~L}^{-1} \mathrm{NaC} 1$ at $\mathrm{pH} 7.2$. Cells were resuspended in distilled water and freeze-dried. The magnetite is pure, nearly stoichiometric $\mathrm{Fe}_{3} \mathrm{O}_{4}$ (Sparks et al., in press). The saturation magnetization $\left(\mathrm{J}_{\mathrm{s}}\right)$ corresponded to $1.5 \%$ magnetite per dry cell weight [Bazylinski et al., 1988]. The freeze-dried powder was used for low temperature measurements and the powder was mixed in a non-magnetic epoxy matrix for room temperature remanence measurements. 
Strain GS-15 was grown under anaerobic conditions as described previously [Lovley and Phillips, 1988]. The copious black precipitate produced during growth, identified as pure $\mathrm{Fe}_{3} \mathrm{O}_{4}$ [Lovley et al., 1987], was freeze-dried and used for measurement. The saturation magnetization corresponded to $56 \%$ magnetite by weight. The sample also included organic debris and growth medium. Freeze-dried powder was mixed in epoxy for room-temperature remanence measurements.

Magnetic measurements were made in fields up to $2.5 \mathrm{~T}$ in the temperature range 2 to $300 \mathrm{~K}$ using a SQUID susceptometer. Room temperature remanence properties were measured using a SQUID magnetometer. Room temperature coercivity spectra [e.g., Cisowski, 1981] were determined by the acquisition of isothermal remanent magnetization (IRM) and by the alternating field (AF) demagnetization of saturation remanent magnetization (SIRM). IRM was given in dc fields up to $100 \mathrm{mT}$ using a short-duration pulse magnetizer, whereas SIRM was given in a steady dc field of $500 \mathrm{mT}$ using an electromagnet.

Mössbauer measurements on whole cells of MV-1 and the black precipitate produced by GS-15 were made with a constant acceleration Mössbauer spectrometer with a variable temperature dewar.

\section{RESULTS}

Room temperature coercivity spectra for MV-1 and GS-15 are shown in Figures 1 and 2 . The results for MV-1 show almost symmetrical behavior between IRM acquisition and SIRM demagnetization (crossover point $\mathrm{R}=0.48$ ). This behavior is consistent with that of an ensemble of nearly noninteracting SD grains [e.g., Cisowski, 1981]. It is similar to results reported for other whole cell magnetotactic bacteria [Stolz et al., 1986; Moskowitz et al., 1988]. However, all estimates of coercivity for MV-1 are higher than those reported for MS-1 [Moskowitz et al., 1988]. Results are summarized in Table 1. The higher coercivities for MV-1 are likely due to the elongation of the individual particle in MV-1 because 
chain length is roughly the same for both MV-1 and MS-1 (10-20 particlesfbacterium). We also observe that measured values of remanent coercivities $\left(H_{r}, H_{r}{ }^{\prime}\right.$, and $\left.R\right)$ are dependent on whether the IRM is given in the presence of a dc field (duration $100 \mathrm{sec}$ ) or in a pulse field (duration $10^{-3} \mathrm{sec}$ ). This effect is related to time dependent phenomena and will be the subject of a future publication.



Fig. 1. Normalized curves of acquisition and AFdemagnetization of SIRM for MV-1.

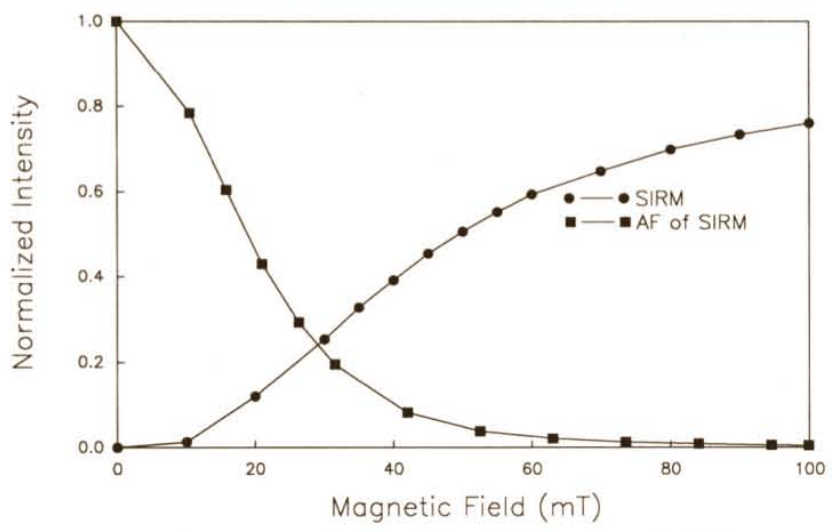

Fig. 2. Normalized curves of acquisition and AF demagnetization of SIRM for GS-15.

Fig. 2. Normalized curves of acquisition and AF demagnetization of SIRM for GS-15.

In contrast, the coercivity spectrum for GS-15 exhibits asymmetric behavior (see Figure 2); it is more difficult to magnetize than to demagnetize $(\mathrm{R}-0.25)$. This behavior is consistent with the effects of magnetostatic interactions resulting from particle agglomeration [Cisowski, 1981]. The coercivity spectra for GS-15 is similar to that reported by Moskowitz et al. [1988] for a freeze-dried samples of MS-1 
consisting of magnetosome chains separated from cells (see Table 1). In both cases, magnetostatic interactions are responsible for the offsets in the coercivity spectra. These results show that room temperature coercivity spectra alone cannot reliably distinguish between magnetite produced by magnetotactic and by dissimilatory iron-reducing bacteria.

\begin{tabular}{|c|c|c|c|c|}
\hline Parameter & MV - 1 & GS -15 & MS - $1 a$ & MS - $1 b$ \\
\hline $\mathrm{H}_{\mathrm{C}}$ & 28.5 & 0.8 & 26.7 & 3.7 \\
\hline $\mathrm{H}_{\mathrm{r}}$ & 48.5 & 30.0 & 37.8 & 24.1 \\
\hline $\mathrm{H}_{\mathrm{r}}^{2}$ & 49.7 & 50.0 & 38.8 & 31.2 \\
\hline $\mathrm{MDF}$ & 49.2 & 18.9 & 37.8 & 10.9 \\
\hline $\mathrm{R}$ & 0.48 & 0.25 & 0.48 & 0.17 \\
\hline $\mathrm{SIRM} / \mathrm{J}_{\mathrm{S}}$ & 0.48 & 0.02 & 0.53 & 0.41 \\
\hline $\mathrm{H}_{\mathrm{r}} / \mathrm{H}_{\mathrm{c}}$ & 1.70 & 37.5 & 1.42 & 6.51 \\
\hline
\end{tabular}

Note: $\mathrm{H}_{c}=$ coercive force, $\mathrm{H}_{1}=$ coercivity of remanence, $\mathrm{H}_{r}{ }^{\prime}=$ median dc field for SIRM acqusition, MDF-median AF destructive field for SIRM. Units are in milltesla $(m T) . H_{r}, H_{r}{ }_{r}$, and $R$ are determined from pulse field experiments. Ms-1a refers to sample consisting of whole cells and Ms-1b refers to sample consisting of magnetosomes chains separated from cells. Steady field measurements for Ms-1a,b are given in Moskowitz et al. [1988].

The thermal decay of a low temperature SIRM for both samples are compared in Figure 3.

Samples of MV-1 and GS-15 were cooled to $20 \mathrm{~K}$ and $2 \mathrm{~K}$ respectively in zero field. Then an isothermal remanence was given in $2.5 \mathrm{~T}$. The field was reduced to approximately zero, and the remanence was measured as the temperature was gradually increased to room temperature. Magnetite produced by MV-1 displayed a sharp discontinuity in intensity at $100 \mathrm{~K}$, approximately $20 \mathrm{~K}$ below the Verwey transition of bulk magnetite. Below and above the transition IRM decreased slowly with increasing temperature. The transition produced a 35\% decrease in intensity by $140 \mathrm{~K}$. Magnetite produced by GS15 displayed a gradual decrease in intensity with temperature and by $140 \mathrm{~K}$ roughly $68 \%$ of the initial remanence had decayed.The thermal decay of a low temperature SIRM is therefore sufficient to distinguish between magnetite produced by MV-1 and GS-15.

The Verwey transition in MV-I magnetite was clearly seen in the M6ssbauer spectra between 
$100 \mathrm{~K}$ and $115 \mathrm{~K}$ (Figure 4). No evidence of a Verwey transition was seen in the GS-15 spectra (data not shown).

The marked difference in low temperature behavior between MV-1 and GS-15 is due to differences in grain-size distributions. At room temperature, the superparamagnetic (SPM) to SD transition for magnetite occurs around 20-40 nm [Dunlop, 1973]. MV-1 contains chains of SD particles that are within the stable SD size range for magnetite at 'room temperature. The gradual decrease in intensity exhibited by GS-15 is caused by the progressive unblocking of magnetization, starting as low as $2 \mathrm{~K}$. This behavior suggests that the grain size distribution in GS-15 extends well into the SPM region for magnetite. In addition, $\mathrm{SIRM} / \mathrm{J}_{\mathrm{s}}$ decreases by $91 \%$ and $\mathrm{H}_{\mathrm{c}}$ decreases by $94 \%$ between $50 \mathrm{~K}$ and $300 \mathrm{~K}$. The decrease in both ratios with increasing temperature is consistent with SPM behavior. However, the finite values of coercivity and remanence and the large value of $\mathrm{H}_{r} / \mathrm{H}_{c}$ (see table 1 ) at $300 \mathrm{~K}$ shows that there is an admixture of SPM and SD particles. The magnetic results are consistent with grain size distributions obtained with high-resolution transmission electron microscope measurements on MV-I and GS15 magnetites (Sparks et al., in press).

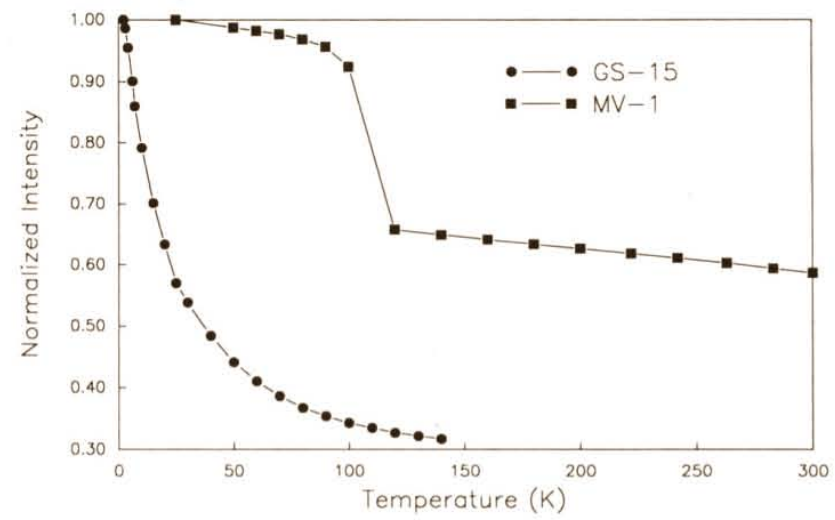

Fig. 3. Thermal decay of saturation remanence for MV-1 and GS-15. SIRM was applied in $2.5 \mathrm{~T}$ field at $20 \mathrm{~K}$ for MV-I and $2 \mathrm{~K}$ for GS-15.

Estimates of the mean particle size $\left(D_{v}\right)$ in GS-15 can be determined magnetically using the room temperature magnetization curve. This method assumes a lognormal distribution of SPM particle 
sizes and requires low-field susceptibility and high-field magnetization data [Chantrell et al., 1978]. The room temperature data give a mean particle diameter of $9 \mathrm{~nm}$. The value of SIRM/J $\mathrm{s}-0.02$ at $300 \mathrm{~K}$ suggests that approximately $96 \%$ of the particle diameters are less than the SPM threshold size. The percentage decreases to $60 \%$ by $50 \mathrm{~K}$. The estimate of the SPM fraction is a maximum because interactions can lower the value of SIRM/J s independent of thermal fluctuations.



Fig. 4. Mossbauer spectra of cells of MV-1 at $120 \mathrm{~K}$ (top), $105 \mathrm{~K}$ (center), and $90 \mathrm{~K}$ (bottom) during cooling from $140 \mathrm{~K}$.

Once $D_{v}$ is known, an average uniaxial anisotropy constant for GS-15 can be estimated from the low temperature remanence data (Figure 3). If the mean unblocking temperature $\left(T_{b}\right)$ is taken as the temperature at which remanence has decayed by half, $D_{v}$ becomes equal to the SPM diameter $\left(D_{s}\right)$ This occurs when $\pi \mathrm{K}_{\mathrm{u}} \mathrm{D}_{\mathrm{v}}{ }^{3}=150 \mathrm{k} \mathrm{T}_{\mathrm{b}}$, where $\mathrm{Ku}$ is the uniaxial anisotropy constant and $\mathrm{k}$ is Boltzmann constant. Here we have assumed that particles are spherical, the dc measurement time is one minute, and the frequency factor is $109 \mathrm{~s}-1$. Using Dv determined from the magnetization data at $300 \mathrm{~K}$ and $\mathrm{Tb}=35 \mathrm{~K}$, we calculate that Kur3.3x105 ergs cm-3. This value is higher than the magnetocrystalline constant and suggests that shape anisotropy is predominant. Chaining of particles due to magnetostatic interactions 
may also contribute to $\mathrm{K}_{\mathrm{u}}$. Our estimate of $\mathrm{K}_{\mathrm{u}}$ agrees with results obtained on magnetite ferrofluids with similar mean particle sizes [Soffge and Schmidbauer, 1981].

Finally, once $\mathrm{K}_{\mathrm{u}}$ is known the critical size for SPM behavior at any temperature can be calculated. From the data in Figure 3, the minimum blocking temperature of $2 \mathrm{~K}$ yields a SPM size of $4 \mathrm{~nm}$, whereas at $300 \mathrm{~K}$ the SPM size is $19 \mathrm{~nm}$.

\section{CONCLUSIONS}

We have shown that the thermal decay of low, temperature SIRM is sufficient to distinguish between magnetite produced by magnetotactic bacteria MV-1 and by dissimilatory iron-reducing bacteria GS-15. The contrast in low temperature magnetic properties is due exclusively to different grain size distributions produced by MV-I and GS15. Magnetotactic bacteria synthesize magnetite with a very narrow grain size distribution that is within the stable SD size range at room temperature and below. The step-like change in remanence at $100 \mathrm{~K}$ exhibited by MV-I is related to the isotropic point in magnetite where the magnetocrystalline anisotropy becomes zero. However, the transition temperature observed for MV-1 is depressed by roughly $20 \mathrm{~K}$ from its value in bulk magnetite. The lower transition temperature is probably due to the fine-grain size of the MV-1 magnetite [Nagata et al., 1964]. Our results suggest that a combination of magnetocrystalline and shape anisotropy controls the remanence in the chain of particles in MV-1. The magnetocrystalline contribution is surprising and most interesting considering that the individual particles are elongated and that a chain of particles also produces an additional effective shape anisotropy. A different batch of MV-I showed similar behavior and therefore rules out any possibility of a mutant strain. In contrast, preliminary results from MS-1 indicates that the low temperature remanence transition is suppressed. A detailed study of the lowtemperature properties of MV-1 and MS-1 will be published separately. 
Unlike MV-1, GS-15 produces magnetite with a broad grain size distribution extending well into the SPM size range for magnetite. The gradual decrease of remanence with temperature, starting as low as $2 \mathrm{~K}$, and the absence of the Verwey transition, confirm that GS-15 produces mostly SPM magnetite. An average particle size of $9 \mathrm{~nm}$ was determined from room temperature magnetization data with some particles as small as $4 \mathrm{~nm}$. However, GS-15 does produce some particles that are stable SD (35-40 nm) because of the finite values of coercivity and remanence observed at room temperature (Figure 2). These particles, unlike the SPM ones, could contribute to the remanence carrying magnetic fraction in suboxic sediments.

Acknowledgments. We thank J. Kirschvink for re-measuring the pulse-field coercivity spectra for MS-1I and R. Sheldon (UCD Physics Dept.) for allowing use of the susceptometer. BMM and RBF were supported by the National Science Foundation. DAB and HWJ were supported by NSF grant OCE87-58I. This is contribution no. 6989 of the Woods Hole Oceanographic Institution.

\section{REFERENCES}

Bazylinski, D.A., et al., Anaerobic magnetic production by a marine, magnetotactic bacterium, $\underline{\text { Nature }}$, 334, 518-519, 1988.

Blakemore, R.P., Magnetotactic bacteria, Annu. Rev. Microbiol., 36, 217-238, 1982.

Blakemore, R.P., et al., Isolation and pure culture of a freshwater magnetic spirillivrn in Chemically defined medium, J. Bacteriol., 140, 720-729, 1979.

Blakemore, R.P., et al., Microaerobic conditions are required for magnetite formation within Aguaspirillum magnetotacticum, Geomicrobiol. J., 4, 53-71, 1985.

Chantrell, R.W., et al., Measurements of particle size distribution parameters in ferrofluids, IEEE Trans Magn., Mag-14, 975-977, 1978.

Cisowski, S., Interacting vs. non-interacting single domain behavior in natural and synthetic samples, Phys. Earth Planet. Inter., 26, 56-62, 1981.

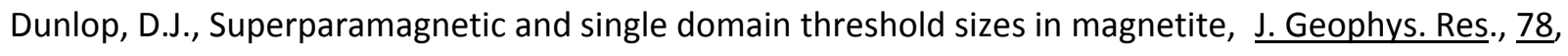
1780-1793, 1973.

Frankel, R.B., et al., Magnetite in freshwater magnetotactic bacteria, Science, 203, 13551356, 1979. 
Karlin, R., et al., Authigenic magnetite formation in suboxic marine sediments, $\underline{\text { Nature, }} \underline{326}, 490-493$, 1987.

Lovley, D., and E.J.P. Phillips, Novel mode of microbial energy metabolism: organic carbonoxidation coupled to dissimilatory reduction of iron or manganese, Appl. Environ. Microbiol., 54, 1472-1480, 1988.

Lovley, D., et al., Anaerobic production of magnetite by a dissimilatory iron-reducing microorganism, Nature, $\underline{330}, 252-254,1987$.

Moskowitz, B.M., et al., Magnetic properties of magnetotactic bacteria, J. Magn. Magn. Mat., $\underline{73}$, 273288, 1988.

Nagata, T., et al., Identification of magnetite and hematite in rocks by magnetic observation at low temperature, J. Geophy. Res., 69, 21112120, 1964.

Petersen, N., et al., Fossil bacterial magnetite in deep-sea sediments from the South Atlantic Ocean, Nature, $\underline{320}, 611-615,1986$.

Soffge, F., and E. Schmidbauer, AC susceptibility and static magnetic properties of Fe304 ferrofluid, $\underline{\mathrm{J}}$. Magn. Magn. Mat., 24, 54-66.

Stolz, J.F., et al., Magnetotactic bacteria and single-domain magnetite in hemipelagic sediments, Nature, 321, 849-851, 1986.

Vali, H., et al., Magnetotactic bacteria and their magnetofossils in sediments, Earth Planet. Sci. Lett., $\underline{86}$, 389-400, 1987. 